\title{
Diminuição da ocorrência de pneumotórax em recém-nascidos com síndrome de desconforto respiratório através de estratégias de redução de parâmetros ventilatórios
}

\author{
Decrease of the occurrence of pneumothorax in newborns with \\ respiratory distress syndrome through reduction of ventilatory parameters
}

\author{
Marisa A.A. Brunherotti ${ }^{1}$, Jacqueline R. Freitas Vianna ${ }^{2}$, Carmem S.T. Silveira ${ }^{3}$
}

\section{Resumo}

Objetivo: verificar, através de uma estratégia de redução dos parâmetros ventilatórios, o declínio da ocorrência de pneumotórax em recém-nascidos com síndrome do desconforto respiratório.

Método: estudo longitudinal, prospectivo, em recém-nascido pré-termo com síndrome do desconforto respiratório, desenvolvido no Centro de Terapia Intensiva Infantil da Santa Casa de Misericórdia de Franca, no período de julho de 1999 a junho de 2000. Estudouse 127 recém-nascidos com idade gestacional de 25 a 37 semanas, de ambos os sexos, e peso ao nascimento de $625 \mathrm{~g}$ a $2.500 \mathrm{~g}$. Foram analisados parâmetros ventilatórios durante a assistência ventilatória mecânica, em dois grupos de pacientes, um controle e outro em que foram reduzidos os parâmetros ventilatórios, investigando e comparando a incidência de pneumotórax. A existência de associação entre os dados foi verificada através do teste de qui-quadrado, ao nível de significância $\alpha=0,05$ e do teste $t$ de Student, ao nível de significância $\alpha=0,05$ e 0,01 (Sokal \& Rolph,1981).

Resultados: os resultados mostraram que menores parâmetros de fluxo inspiratório, tempo inspiratório, gerando menor volume corrente, diminuem a ocorrência de pneumotórax $(8,1 \%)$, comparado a $(24,5 \%)$ do grupo controle, com significância estatística $\left(\chi^{2}=\right.$ $6,545 ; \mathrm{p}=0,05)$. Não houve significância quanto à mortalidade $\left(\chi^{2}=\right.$ 0,736; $\mathrm{p}=0,391)$.

Conclusão: este estudo mostrou que a utilização de menor tempo inspiratório e fluxo inspiratório em recém-nascidos com síndrome do desconforto respiratório está associada com menor ocorrência de pneumotórax, porém não relacionada com redução da mortalidade.

J Pediatr (Rio J) 2003;79(1):75-80: pneumotórax, recém-nascido, síndrome do desconforto respiratório, estratégia protetora de ventilação pulmonar.

\begin{abstract}
Objective: to verify if the strategy of reduction of the ventilatory parameters decreases the occurrence of pneumothorax in preterm newborns with respiratory distress syndrome.

Methods: a longitudinal prospective study of preterm newborns with respiratory distress syndrome was carried out at the Pediatric Intensive Care Center of Hospital Santa Casa de Misericórdia of Franca, from July 1999 to June 2000. One hundred and twenty-seven female and male newborns between the $25^{\text {th }}$ and the $37^{\text {th }}$ week of gestation, with birthweight varying from $625 \mathrm{~g}$ to $2500 \mathrm{~g}$ were studied. The ventilatory parameters were analyzed during the assisted mechanical ventilation in two groups of patients: the controlled group and the other group, in which the parameters were reduced, by investigating and comparing the occurrence of pneumothorax. The association of data was verified through chi-square test; level of significance $\alpha=0.05$ and 0.01 , and the difference among the studied parameters was obtained through the Student's $t$ test.

Results: the results show lower parameters of inhalation flow and shorter period of inhalation, generating minor tidal volume, decrease of the occurrence of pneumothorax (8.1\% compared to $24.5 \%$ of the controlled group), with statistical significance $\left(\chi^{2}=\right.$ $6.545 ; \mathrm{p}<0.05)$, and without significance related to mortality and hospital discharge $\left(\chi^{2}=0.736 ; \mathrm{p}=0.391\right)$.

Conclusions: this study verified that lower inhalation flow and shorter period of inhalation in preterm newborns with respiratory distress syndrome were associated with the decrease of the occurrence of pneumothorax.
\end{abstract}

J Pediatr (Rio J) 2003;79(1):75-80: pneumothorax, preterm newborn, respiratory distress syndrome, protective ventilation strategy.

1. Fisioterapeuta especialista em pneumologia funcional e psicossomática. Fisioterapeuta responsável do Centro de Terapia Intensiva Infantil do HSCM de Franca, SP. Docente do Curso de Graduação e Pós-graduação em Fisioterapia da UNIFRAN - Franca, SP.

2. Fisioterapeuta especialista em pneumologia funcional. Fisioterapeuta responsável do Centro de Reabilitação Respiratória - Franca, SP. Docente do Curso de Graduação e Pós-graduação -UNIFRAN- Franca, SP.

3. Título de Especialista em Pediatria, Neomatologia e Terapia Intensiva Pediátrica, Médica Plantonista do C.T.I. Infantil da Santa Casa e da U.T.I. Neonatal da UNIMED - Franca, SP. 
O sistema respiratório do recém-nascido $(\mathrm{RN})$ apresenta algumas particularidades que o tornam mais suscetível à necessidade do suporte ventilatório ${ }^{1}$. Alguns fatores anatômicos e fisiológicos, como vias aéreas de menor calibre, tórax cilíndrico, costelas horizontalizadas, grande quantidade de fibras musculares tipo IIa, predominância do sono REM e imaturidade do sistema nervoso central (SNC) proporcionam uma ineficiência na mecânica respiratória, favorecendo a distorção torácica, com maior incidência de fadiga muscular respiratória e colapso pulmonar, tornandoo mais susceptível à necessidade de ventilação mecâni$\mathrm{ca}^{2-6}$.

Em vista da grande incidência das complicações relacionadas à ventilação mecânica, surgiu o interesse em estudar a estratégia terapêutica para minimizar os riscos potenciais, como o pneumotórax (PTX).

A ventilação mecânica $(\mathrm{VM})$ nos recém-nascidos com síndrome do desconforto respiratório (SDR) tinha, como principal objetivo, a manutenção dos gases sanguíneos normais, através da utilização de altos volumes correntes, com conseqüentes picos e pressões inspiratórios altos, aumentando o risco de pneumotórax ${ }^{7}$. Estes picos de pressões elevados levam a uma lesão pulmonar induzida pela ventilação mecânica, que se caracteriza por dano no parênquima pulmonar, agravando a injúria histológica da SDR, assim propiciando o pneumotórax ${ }^{8}$.

Esta lesão da membrana alvéolo-capilar, pré-existente na SDR, e que ocorre também em pacientes submetidos à VM, é melhor definida como lesão pulmonar associada à ventilação mecânica ${ }^{9}$.

Estudos clínicos verificaram diferentes estratégias protetoras pulmonares através de redução dos parâmetros ventilatórios, como diminuição de volume corrente e de pico de pressão inspiratória. Alguns desses estudos sugerem efeitos benéficos, como redução da lesão parenquimatosa e da mortalidade ${ }^{10,11}$.

Devido às dificuldades em se determinar as pressões alveolares em modelos clínicos, as pressões de vias aéreas têm sido utilizadas como indicadores de risco para a ocorrência de escape de ar extra-alveolar ${ }^{12}$.

Entretanto, existem algumas limitações em se utilizar as pressões proximais de vias aéreas para determinar o risco para o barotrauma, pois estas pressões proximais podem não refletir as pressões alcançadas ao nível alveolar, que são afetadas por mudanças na resistência e fluxo ${ }^{13}$. Outros fatores, como excessivas variações nos volumes pulmonares, com conseqüente superdistensão alveolar, também poderia ser determinante primário da lesão pulmonar iatrogênica, denominado volutrauma ${ }^{14}$.

Diante desta dificuldade em se estabelecer parâmetros ideais durante a assistência ventilatória mecânica, esta pesquisa tem o objetivo de verificar uma estratégia de redução dos parâmetros ventilatórios para diminuir a inci- dência de pneumotórax em recém-nascidos com síndrome do desconforto respiratório na Unidade de Terapia Intensiva da Santa Casa de Misericórdia de Franca.

\section{Casuística e métodos}

Realizou-se estudo longitudinal, comparativo, de 127 recém-nascidos pré-termo ( $<37$ semanas, WHO, 1974), sendo 52 pacientes do sexo feminino $(41 \%)$ e 75 do sexo masculino (59\%). O peso ao nascimento destes recémnascidos pré-termo (RNPT) variava entre $625 \mathrm{~g}$ a $2.500 \mathrm{~g}$, e a idade gestacional variava de 25 semanas a 37 semanas, segundo o método Capurro ${ }^{15}$. O estudo foi aprovado pelo Comitê de Ética da Santa Casa de Misericórdia de Franca.

Os RNPT do nosso estudo foram diagnosticados com SDR de acordo com os critérios clínicos e radiológicos e gasométricos. Os critérios clínicos foram seguidos de aumento da freqüência respiratória, cianose persistente, escore Silverman-Anderson maior ou igual a 7 pontos, raios-X com imagem de padrão reticulogranular difuso em ambos os pulmões, com broncogramas aéreos de intensidade variável, e valores gasométricos com $\mathrm{PaO}_{2}<50 \mathrm{mmHg}, \mathrm{PaCO}_{2}>$ $60 \mathrm{mmHg}$ e $\mathrm{PH}<7,25^{1}$.

Os critérios de inclusão foram RN menor que 37 semanas de gestação e peso menor que $2.500 \mathrm{~g}$, e excluímos os recém-nascidos maiores que 37 semanas de gestação com peso acima de $2.500 \mathrm{~g}$ e anomalias.

Foram usados respiradores Intermed e Shchrist, de fluxo contínuo, ciclados a tempo e limitados à pressão, controlados por microprocessadores e com circuitos eletrônicos independentes para alarme e monitorização de pressão. Os parâmetros eram modificados, usando como critério as alterações clínicas dos RN. Estas alterações eram aleatórias e pessoais, em cada plantão.

Os recém-nascidos foram estudados em 2 coortes controladas, desde os primeiros dias de vida. O grupo "A" (grupo histórico), com 53 pacientes, foi analisado no período de junho a dezembro de 1999, e o grupo "B" (estratégia de redução de parâmetros ventilatórios), com 74 pacientes, de janeiro a junho de 2000. As análises foram feitas através de coleta de dados, em uma ficha controle, com os seguintes dados: nome, data de nascimento, internação, sexo, tipo de parto, peso, parâmetros do respirador, exames complementares, uso de surfactante e incidência de pneumotórax. Estes dados foram colhidos todos os dias, no período da manhã, em um mesmo horário, excluindo o domingo, durante o período de assistência ventilatória mecânica.

Os dois grupos diferem entre si pelas modificações dos parâmetros da ventilação mecânica, que foram alterados na tentativa de uma estratégia de redução do pneumotórax ocorrido no primeiro grupo. Os parâmetros modificados foram fluxo (VI) e o tempo inspiratório (TI), na tentativa de obter um menor volume corrente (VC). A média dos parâmetros foi obtida no período de 1 a 5 dias de ventilação mecânica. 
O VI e o TI são ajustados nos respiradores ciclados a tempo, e o produto destes parâmetros determina o VC. Assim, o VC $=$ TI $x$ VI $(\mathrm{VC}=\mathrm{ml} ; \mathrm{TI}=$ seg e $\mathrm{VI}=$ $\mathrm{ml} / \mathrm{seg})^{16,17}$.

O TI depende da constante de tempo, e vai variar de acordo com a complacência pulmonar. O VI habitualmente utilizado é um valor 3 vezes maior que o volume minuto (VM). O VC depende da complacência pulmonar, do gradiente de pressão (Pinsp - PEEP); em geral, utiliza-se VC de 6 a $8 \mathrm{ml} / \mathrm{kg}$. O uso de $\mathrm{VC}$ altos ( 10 a $12 \mathrm{ml} / \mathrm{kg}$ ) pode resultar em hiperdistensão alveolar e risco de síndrome de escape de ar ${ }^{18,19}$.

Aa pressões médias de vias aéreas foram obtidas através da equação expressa por:

\section{MAP = k (PIP-PEEP) [TI/(TI+TE) $]$ + PEEP1.}

O pneumotórax foi diagnosticado através de manifestações clínicas e confirmação radiológica. O RN pode apresentar-se assintomático, ou mostrar graus variáveis de desconforto respiratório, como gemido, taquipnéia e retrações. Há piora súbita da insuficiência respiratória, agitação, cianose e queda da saturação do oxigênio. À inspeção do tórax, o lado comprometido pode apresentar-se abaulado; à ausculta, o murmúrio vesicular está diminuído. As bulhas cardíacas podem estar abafadas, podendo ocorrer tamponamento cardíaco, hipotensão e choque. O fígado e o baço podem ser palpáveis devido ao rebaixamento do diafragma. Se o diagnóstico precoce não foi realizado, poderá ocorrer o óbito ${ }^{20,21}$.

Os resultados obtidos foram submetidos à análise estatística utilizando-se os testes de qui-quadrado ao nível de significância $\alpha=0,05$ e teste $t$ de Student, ao nível de significância $\alpha=0,05$ e 0,01 (Sokal \& Rolph, 1981) 22 .

\section{Resultados}

Foram incluídos no estudo 127 RNPT, sendo 53 pacientes no grupo A (controle), que apresentaram média de peso de $1.395 \mathrm{~g}$ e desvio padrão de $493,6 \mathrm{~g}$, e 74 pacientes no grupo B, no qual se reduziu os parâmetros ventilatórios, e que apresentaram média de peso $1.547 \mathrm{~g}$ e desvio padrão de $510 \mathrm{~g}$.

A distribuição dos pacientes nos grupos, com relação a sexo, à idade gestacional e peso ao nascimento foi homogênea.

No grupo A (54 pacientes), houve uma incidência de 13 pneumotórax (24,5\%), e no grupo B (74 pacientes,) a incidência foi de 6 pneumotórax $(8,1 \%)$, obtendo uma significância $(\mathrm{p}<0,05)$ na comparação entre os grupos (Tabela 1).

A comparação entre os grupos não apresentou uma significância estatística. O grupo A apresentou 50,9\% de óbito e $49,1 \%$ de alta hospitalar, e o grupo B apresentou $43,2 \%$ de óbito e $56,8 \%$ de alta hospitalar $(\mathrm{p}=0,391)$ (Tabela 1).

Os pacientes do grupo A que apresentaram pneumotórax tinham peso médio de $1.203 \mathrm{~g}$, e receberam uma média de pressão positiva no final da expiração (PEEP) de 6,26 $\mathrm{cmH}_{2} \mathrm{O}$, PI de 21,87 $\mathrm{cmH}_{2} \mathrm{O}$, Fr de 30,30 ciclos/minuto, TI de 0,64 seg, fluxo inspiratório de 8,261/m, e MAP de 11,57. Os do grupo B tinham peso médio de $1.192 \mathrm{~g}$ e receberam uma média de PEEP de $6,11 \mathrm{cmH}_{2} \mathrm{O}$, uma PI média de 21,8 $\mathrm{cmH}_{2} \mathrm{O}$, Fr de 28,12 cilos /minuto, TI médio de 0,637 seg, fluxo inspiratório de 6,38 1/m, e MAP de 10,38. Na comparação entre os grupos, apresentaram diferença significativa o TI e a VI $(\mathrm{p}<0,01)$, e a MAP $(\mathrm{p}<0,05)$. Os outros parâmetros não apresentaram diferença significante (Tabela 2).

Tabela 1 - Comparação da incidência de pneumotórax e destino dos pacientes do grupo A e do grupo B

\begin{tabular}{|c|c|c|c|c|c|c|c|c|}
\hline \multirow[b]{3}{*}{ Desfecho } & \multicolumn{4}{|c|}{ Incidência de pneumotórax } & \multicolumn{4}{|c|}{ Óbito } \\
\hline & \multicolumn{2}{|c|}{ Grupo A } & \multicolumn{2}{|c|}{ Grupo B } & \multicolumn{2}{|c|}{ Grupo A } & \multicolumn{2}{|c|}{ Grupo B } \\
\hline & $\mathbf{N}^{\mathbf{o}}$ & $\%$ & $\mathbf{N}^{\mathbf{o}}$ & $\%$ & $\mathbf{N}^{\mathbf{o}}$ & $\%$ & $\mathrm{~N}^{0}$ & $\%$ \\
\hline Positivo & 13 & 24,5 & $6 *$ & 8,1 & 27 & 50,9 & $32 \dagger$ & 43,2 \\
\hline Negativo & 40 & 75,5 & 68 & 91,9 & 26 & 49,1 & 42 & 56,8 \\
\hline
\end{tabular}

* Significativamente menor que expostos, de acordo com qui-quadrado $\left(\chi^{2}=6,545 ; p<0,05\right)$

† Diferença não significativa $\left(\chi^{2}=0,736 ; p=0,391\right)$ 
Tabela 2 - Comparação entre médias (desvio padrão) do grupo A e do grupo B para parâmetros seguintes

\begin{tabular}{|c|c|c|c|c|c|c|}
\hline \multirow[b]{2}{*}{ Parâmetro } & \multicolumn{2}{|c|}{ Grupo A } & \multicolumn{2}{|c|}{ Grupo B } & \multicolumn{2}{|c|}{ Teste $t$ Students } \\
\hline & Média & DP & Média & DP & $t$ & $\mathbf{p}$ \\
\hline $\mathrm{TI}$ & 0,64 & 0,04 & $0,59^{\dagger}$ & 0,06 & 5,204 & 0,000 \\
\hline Fluxo & 8,26 & 1,21 & $6,38^{\dagger}$ & 1,11 & 9,102 & 0,000 \\
\hline PIP & 21,87 & 3,75 & $21,08 \ddagger$ & 3,16 & 1,280 & 0,203 \\
\hline PEEP & 6,26 & 0,71 & $6,11 \div$ & 0,85 & 1,088 & 0,279 \\
\hline FR & 30,30 & 7,97 & $28,12 \ddagger$ & 7,59 & 1,564 & 0,120 \\
\hline $\mathrm{TE}$ & 1,49 & 0,57 & $1,71 \ddagger$ & 0,77 & $-1,834$ & 0,069 \\
\hline MAP & 11,57 & 2,71 & $10,38 *$ & 2,37 & 2,621 & 0,010 \\
\hline
\end{tabular}

* $\quad(p<0,05)$ e $\dagger(p<0,01)$ de acordo com teste $t$ de Student

‡ diferença não significativa

\section{Discussão e conclusão}

No período neonatal tem-se grande risco para o desenvolvimento de síndrome de escape de ar, pois há uma maior frequiência de falência respiratória nesta idade, implicando o uso de suportes ventilatórios e procedimentos de reanimação neonatal, com a aplicação de pressão positiva.

Ogata et al. relataram incidência variável relacionada ao grau de falência respiratória e ao modo de ventilação utilizado em RNs portadores de SDR. Neste estudo, a incidência de pneumotórax foi de 3,5\% nos RNs que não receberam assistência ventilatória, $11 \%$ nos casos em que se utilizou pressão positiva contínua nas vias aéreas (CPAP), e 33\% nos casos que necessitaram de ventilação mecânica com emprego de pressão expiratória final positiva (PEEP) ${ }^{21}$.

Em estudos retrospectivos envolvendo 119 RNs com SDR, Madansky et al. encontraram alguma forma de escape de ar em 27\% dos RNs. Destes, $24 \%$ desenvolveram o escape de ar durante tratamento com oxigênio inalatório, $16 \%$ durante o uso de CPAP, e 34\% na vigência de ventilação mandatória intermitente. Dentre os RNs com diagnóstico de SAM, 41 apresentaram alguma forma de escape de ar, enquanto nos portadores de taquipnéia transitória, a incidência foi de $10 \%^{23}$.

Goldberg e Abdenour citam incidência de $24 \%$ de pneumotórax nos RNs prematuros com SDR em suporte ventilatório, que não receberam surfactante, contra $5,9 \%$ no grupo que recebeu surfactante como terapêutica para a SDR. Outros trabalhos mostram maior incidência de pneumotórax nos RNs que, sob ventilação mecânica, receberam maior pico de pressão inspiratória (PIP), maior tempo inspiratório (TI) e maior pressão média em vias aéreas ${ }^{24,25}$.

Segundo Amato et al., a aplicação de uma estratégia protetora de ventilação pulmonar, utilizando PEEP abaixo do ponto de inflexão da curva pressão/volume, volume corrente de $6 \mathrm{ml} / \mathrm{kg}$ e pressão inspiratória menor que 20 $\mathrm{cmH}_{2} \mathrm{O}$, diminuiu a incidência de barotrauma e aumentou a freqüência de desmame da ventilação mecânica, quando comparada à ventilação mecânica convencional ${ }^{10}$.

No RN, a duração do TI é bem mais curta, assim como o volume inspirado, o que reflete uma complacência específica baixa. Relacionando o VC com o TI, a PI é bem maior nos RN que nos adultos ${ }^{1}$. O uso de PI muito baixas pode ocasionar hipoventilação com hipoxemia e hipercapnia; por outro lado, o uso de PI altas relaciona-se com o aparecimento de síndrome de escape de ar, com o aumento da resistência vascular pulmonar e com doença pulmonar crônica em longo prazo $^{26}$.

O TI depende de uma constante de tempo, que se relaciona diretamente com a complacência pulmonar. Quando a complacência pulmonar é baixa, a constante de tempo também fica menor. Assim, o TI empregado na ventilação poderá ser tão pequeno quanto 0,3 a $0,5 \mathrm{seg}$. Quando a complacência pulmonar se encontra próxima ao normal, a constante de tempo é maior e, conseqüentemente, se farão necessários TI maiores, próximos de 0,5 a $0,7 \mathrm{seg}^{27}$.

O VI tem relação direta com o TI e a PI. Um fluxo alto no respirador $(>61 / \mathrm{m})$ atinge facilmente a pressão predeterminada, porém submete os alvéolos a um pico de pressão por um período de tempo prolongado, fornecendo altos volumes correntes ${ }^{26}$. O alto fluxo também promove um fluxo turbulento que eleva a resistência da via aérea, e isso implica em incremento do trabalho respiratório. O gasto energético para vencer a resistência ao exalar pode ser tão elevado que poderá ocorrer uma PEEP indesejável no final da expiração ${ }^{28}$. Esse tipo de ventilação pode ser eficaz para corrigir a hipoxemia, mas está relacionada com uma alta incidência de lesão pulmonar, como o pneumotórax ${ }^{18}$. 
A incidência de pneumotórax é alta na SDR, quando se empregam altas pressões e/ou altos volumes durante a ventilação mecânica. Amato et al. têm preconizado o valor da aplicação de uma estratégia protetora de ventilação mecânica com o uso de volume corrente e pressão inspiratória baixos, para minimizar a injúria pulmonar, podendo não somente reduzir complicações, como também a mortalidade de pacientes com $\mathrm{SDR}^{29}$.

Os resultados mostram uma maior incidência de pneumotórax $(24,5 \%)$ no grupo que recebeu maior VI e TI, quando comparado ao grupo em que estes parâmetros foram reduzidos $(8,1 \%)$.

As pesquisas mais atuais enfocam estratégias ventilatórias com proteção pulmonar, ou seja, aquelas que recrutam um volume pulmonar adequado, sem hiperdistensão; reduzindo, assim, o aparecimento de pneumotórax ${ }^{11,30}$.

Através deste estudo, verificamos que TI menor que 6 seg e VI menor que 7 1/min, na fase inicial da SDR, são relacionados com uma menor frequiência de pneumotórax; porém esta tentativa de estratégia protetora, com menor fluxo e tempo inspiratório, não está associada com diminuição da mortalidade.

\section{Agradecimentos}

Deixo sinceramente os nossos agradecimentos à equipe médica desta unidade, composta pelos Drs. Alencar, Andréa, Carlos J., Jorge, Lélia, Leda, Luis Peixe, Marcelo, Priscila, Rita e pelo voto de confiança e credibilidade depositado em nosso trabalho.

\section{Referências bibliográficas}

1. Goldsmith JP, Karotkin EH. Introduction to assisted ventilation. In: Goldsmith JP, Karotkin EH, editores. Assisted ventilation of the neonate. $3^{\mathrm{a}}$ ed. Philadelphia: WB Saunders; 1996.p.1-19.

2. Muller NL, Bixan AC. Chest wall mechanics and respiratory muscles in infants. Pediat Clin North Am 1979;26:503-16.

3. Roussos CS, Macklen PT. Diaphragmatic fatigue in man. J App Physiol Repirat. Environ Exercise Physiol 1977;43:189-97.

4. Scarpelli EM, Auld PAM, Goldman HS. Pulmonary disease of the fetres, newborn and child. Philadelphia: Lea \& Febiger; 1978.

5. Kattan M. Long term sequelae of respiratory illness in infancy and childhood. Pediatr Chin North Am 1979;26(3):525-35.

6. Avery ME, Fletcher BD, Willians RG. Hyaline membrane disease. In: Avery ME, Fletcher BD, Willians RG, editores. The cring and its disorder in the newborn infant. Philadelphia: WB Saunders; 1981.

7. Truman TL; Todre ID. Acute respiratory distress syndrome. In: Todres ID, Tugate JH, editores. Critical care of infants and children. Boston: Little Brown; 1996.p.147-159.

8. Slutsky AS. Mechanical ventilation. Chest 1993;104:1833-59.
9. International Consensus Conference Committee:International Consensus Conferece in Intensive care medicine: Ventilator associated lung injury in ARDS. Am J Respir Crit care med 1999;160:2118-24.

10. Amato MB, Barbas CS, Medeiros DM, Magaldi RB, Schettino GP, et al. Effect of a Protective-ventilation Strategy on Mortality in the acute respiratory distress syndrome. N Engl J Med 1998;338(6):347-54.

11. Brochard L, Roudot-Thoraval F, Roupie E, Delclaux C, Chastre J, Fernandez-Mondejar E, et al. Tidal volume reduction for prevention of ventilator-induced lung injiery in acute respiratory distress syndrome. The multicenter trail croup on tidal volume reduction in ARDS. Am J Respir Crit Care Med 1998;158:1831-8.

12. Petersen GW, Baier H. Incidence of pulmonary barotrauma in a medical ICU. Crit Care Med 1983;11:67-9.

13. Gammon RB, Shin MS, Groves RH Jr, Hardin JM, Hsu C, Buchalter SE. Clinical Risk factors for Pulmonary Barotrauma: a multivariate analysis. Am J Respir Crit Care Med 1995;152:1235-40.

14. Dreyfuss D, Saumon G. Role of Tidal Volume, FRC and end expiratory volume in the development of pulmonary edema following mechanical ventilation. Am Rev Respir Dis 1993;1481194-203.

15. Capurro H, Konichezky S, Fonseca D, Caldeyrobarcia R. Simplified method for diagnosis of gestational age in newborn infant. J Pediatric 1978;93(1):120-2.

16. Chatburn RL. Principles and practice of neonatal and pediatric mechanical ventilation. Resp Care1991;36:569-874.

17. Kaiserman KB, Cunningham MD, Martin G, Stevens J. Optimal tidal volume in the neonate during conventional mechanical ventilation. Clinical Research 1992;40(1):A84-A84.

18. Kumar A, Pontoppidan H, Flake KJ, Wilson RS, Laver MB. Pulmonary barotrauma during mechanical ventilation. Cret Care Med 1973;4:181-6.

19. Steier M, Ching N, Roberts EB, Neolon TF. Pneumothorax complicating continuais ventilatory support. J Thorac Cardiac Surg 1974;67:17-23.

20. Krebs VLJ, Trostes EJ. Complicações da ventilação mecânica. Pediatria Moderna 2000;XXXVI:junho (edição especial).

21. Ogata ES, Gregory GA, Kitterman JA, Phibbs RH, Tooley WH. Pneumothorax in the respiratory distress syndrome: incidence and effect on vital signs, blood gases, and $\mathrm{pH}$. Pediatrics 1976;58:177-83.

22. Sokal RR, Rolph FJ. Biometry. $2^{\text {a }}$ ed. San Francisco: WH freemann and Co.; 1981.

23. Madansky DL, Lawson EE, Chernick V, Taeusch HW. Pneumothorax and other forms of pulmonary air leak in newborns. Am Rew Resp Dis 1979;120:729-37.

24. Goldeberg RN, Abdenour GE. Air leak syndrome. In: Spitzer AR, editor. Intensive care of the fetus and neonate. St. Louis: Mosby-yea; 1996.p.629-640.

25. Glenski JA, Hall RT. Neonatal pneumopericardium: analysis of ventilatory variables. Crit Care Med 1984;12:439-42.

26. Spitzer AR, Fox WW. Positive presure ventilation: pressure limited and time - cyded ventilations. In: Goldsmith JP, Karotkin $\mathrm{EH}$, editores. Assisted ventilation of the neonate. $3^{\mathrm{a}}$ ed. Philadelphia: WB Saunders; 1996.p.176-186.

27. Heicher DA, Kasting DS, Harrod JR. Prospective clinical comparison of two methods for mechanical ventilation of neonates: rapid rate and short inspiratory time versus slow rate and long inspiratory time. J Pediatr 1981;98:957-61.

28. Sampietro VI, Azevedo MPO, Resende JG. Medida da resistência do fluxo aéreo em peças nasais de CPAP [site na internet]. Disponível em: http://www.sbp.com.br/jornal/00-03.04/ artarig4.html>. Acessado 11 de novembro de 2001. 
29. Kacmareck RM. Strategies to optimize alveolar recruitment. Crit Care 2001;7:15-20

30. Brower RG, Shanholtz CB, Fessler HE, Shade DM, White P Jr, Wiener CM, et al. Prospective, randomized controlled clinical trial comparing traditional versus reduced tidal volume ventilation in acute respiratory distress syndrome patients. Crit Care Med 1999;27(8):1492-8.
Endereço para correspondência:

Dra. Marisa A.A. Brunherotti

PROENE - Programa de Estudos em Neonatologia

Rua Thomas Gonzaga, 1932 - Centro

CEP 14400-540 - Franca, SP

E-mail: brunherotti@uol.com.br 